\title{
THE EFFECTIVENESS OF VITAMIN D SUPPLEMENTATION IN CHRONIC PERIODONTITIS PATIENTS: A RANDOMIZED CONTROLLED CLINICAL TRIAL
}

\author{
Assaf M.* and Nayer Aboelsaad ${ }^{* *}$
}

\begin{abstract}
Background : Vitamin D deficiency is a common health problem worldwide and linked to some infectious and inflammatory conditions, including periodontal disease. This is a double blind randomized controlled study to evaluate the clinical effectiveness of Vitamin D supplementation as an adjunct therapy to non-surgical periodontal treatment in healthy middle-aged vitamin $\mathrm{D}$ deficient patients with chronic periodontitis.
\end{abstract}

Material and Methods: Twenty eight patients with mild to moderate chronic periodontitis were included in this study. They were randomized to two groups: Test Group taking oral Vitamin D supplementation 10,000 IU one tablet daily for five days per week for a total duration of 12 weeks and Control Group taking placebo pills. Both groups were maintained on scaling and root planning sessions. Data was collected at baseline and at three months including the following clinical periodontal parameters: Gingival index (GI), Plaque index (PI), Probing pocket depth (PPD) and Clinical attachment level (CAL).

Results: Intra-group analysis showed that both groups had significant improvements in the periodontal measures. However, analysis comparing changes in the mean periodontal indices between groups showed that the mean changes across all periodontal indices between groups were statistically significant at $\mathrm{p}<0.05$. The highest significant difference between the test group and the control group was in the probing pocket depth, where the test group had a $26.07 \%$ greater decrease than the control group.

Conclusion: Adjunctive low cost Vitamin D supplementation in mild to moderate chronic periodontitis in middle aged Vitamin D deficient healthy patients significantly improves clinical periodontal outcomes relative to scaling and root planning alone with no side effects. Vitamin $\mathrm{D}$ is safe and probably may contribute to decrease health care costs as an adjunctive therapy to conventional periodontal treatment.

KEY WORDS: Chronic periodontitis, vitamin D deficiency, periodontal diseases/drug therapy, calcitriol/therapeutic use

* Oral Surgical Sciences Department, Division of Periodontology, Faculty of Dentistry, Beirut Arab University, Lebanon ** Associate Professor, Periodontology, Faculty of Dentistry, Beirut Arab University, Lebanon; and Faculty of Dentistry, Mansoura University, Egypt. 


\section{INTRODUCTION}

Periodontitis is a chronic inflammatory disease of which the mild-to-moderate form is the most common, with prevalence estimates ranging from 8 to $14 \%$ among adults in Lebanon. The chronic inflammation, related to underlying oral bacterial infection, can lead to periodontal disease and bone destruction $^{(1,2)}$.

Vitamin D is implicated as a potential antiinflammatory and anti-microbial agent that could modify risk for periodontal disease and subsequent tissue destruction and alveolar bone loss, by altering the individual's immune response to oral bacterial infection and associated inflammatory response. Furthermore, the immunomodulatory effect of vitamin $\mathrm{D}$ has been related to modulation of bacterial-mediated infections, with low levels of vitamin $\mathrm{D}$ being linked with an increased risk of infectious diseases $(3,4)$

There is a high prevalence of vitamin D deficiency in the Eastern Mediterranean Region ${ }^{(5)}$. Meanwhile, studies have shown that low plasma levels of $25(\mathrm{OH})$ Vitamin D were associated with worse periodontal clinical measures such as higher clinical attachment loss and probing pocket depth.However, higher serum concentration of $25(\mathrm{OH})$ Vitamin D was shown to predict a lesser likelihood to bleed on gingival probing which suggests that Vitamin D may decrease susceptibility to gingival inflammation through its anti-inflammatory effects ${ }^{(6,7)}$

In a recent study evaluating the effect of vitamin $\mathrm{D}$ and calcium supplementation in reducing gingival inflammation, a combined daily dose of $500 \mathrm{mg}$ calcium and $250 \mathrm{IU}$ Vitamin D was given to a test group for three months in addition to scaling and root planing. It was shown to improve clinical measures that included the Oral Hygiene IndexSimplified, Gingival Index, and Bone Density $(\mathrm{p}<0.05)$ as compared to scaling and root planing alone $^{(8)}$.
Vitamin D alone may prove to be an adjunct therapy, in addition to the traditional scaling and root planning therapy, for the treatment of periodontal disease and improving oral clinical outcomes. As it was reported that vitamin D increases antibacterial defense of gingival epithelial cells and improves postoperative wound healing after periodontal surgery.

To our current knowledge, there is no randomized controlled double blinded trial reported in the literature to test the effectiveness of Vitamin D supplementation alone on clinical periodontal outcomes.

Therefore, the aim of this randomized controlled trial was to evaluate the clinical effectiveness of Vitamin D supplementation as an adjunct therapy to non-surgical periodontal treatment in healthy middle- aged patients with mild to moderate chronic periodontitis, using several periodontal parameters.

\section{METHOD}

\section{Study Design}

This is a double blinded randomized controlled clinical study.

For ethical requirements, the procedure was explained and a written informed consent was obtained from each patient prior to enrollment in the study. The study was approved by the Institutional Review Board at Beirut Arab University- Lebanon with IRB Number 2014H/0010/D/M/0035.

\section{Patient selection}

Twenty eight chronic periodontitis patients were selected from the periodontology clinic of the Faculty of Dentistry at Beirut Arab University according to the following criteria:

\section{Inclusion criteria}

All patients were selected according to the criteria of the 2017 classification system of 
periodontal diseases and conditions having mild to moderate chronic periodontitis ${ }^{(9)}$. Those patients are healthy, between 30 and 45 years of age, of both genders have at least 20 teeth, are smokers (less than 10 cigarettes a day) and have Vitamin D blood levels below $30 \mathrm{ng} / \mathrm{ml}$ (Normal level is $\geq 30 \mathrm{ng} / \mathrm{ml}$ ).

\section{Exclusion Criteria}

Patients were excluded if they were on: current antibiotic therapy, intake of Vitamin D supplements, corticosteroid or immunosuppressive medications, bisphosphonates or hormonal therapy. Moreover, patients who were at menopause, pregnant, have a history of infective endocarditis, congenital or acquired cardiac defects; cardiac prosthesis, hematological disorders; immune defects, history of diabetes, calcium deficiency, bone diseases or any systemic conditions that might affect the periodontium and the treatment protocol were also excluded.

\section{Protocol}

Patients were randomly allocated to each group using an online random group generator (https:// www.randomizer.org/) provided by an independent third party:

The test group consisted of fourteen patients of both genders. They are treated with scaling and root planing in addition to oral Vitamin D3 supplementation 10,000 IU (Maxi-D® 10,000 I.U. Made by GREENMADE, Lebanon) (Figure 1) one tablet daily for five days per week (Monday to Friday) for a total duration of 12 weeks.

The control group consisted of fourteen patients of both genders. They received a similar treatment in addition to similar looking placebo pills and bottles / one tablet daily for five days per week (Monday to Friday) for a total duration of 12 weeks.

In both groups, scaling and root planing were performed with hand curettes and ultrasonic scalers until all teeth surfaces are clean, hard and smooth as judged by the clinician.(Figures 2,3,4,5)
The researchers and patients were both blinded to the treatment allocation (Vitamin D versus placebo pills) and only after the study protocol was completed, the treatment allocations were disclosed by the independent third party.

\section{Evaluation Phase}

For the Test group, clinical parameters and blood analysis were collected at baseline then after three months period following treatment.

For the Control Group, clinical parameters and blood analysis were collected at baseline and only clinical parameters are reassessed after three months.

As part of the evaluation phase and to ensure patient compliance to the treatment protocol, patients were called once a week during the first month and every two weeks in the second and third month and reminded of the hygiene instructions and of taking the oral supplements as per protocol.

\section{Periodontal Clinical Parameters Measured:}

1- Gingival index (GI)

2- Plaque index (PI)

3- Probing pocket depth (PPD)

4- Clinical attachment level (CAL)

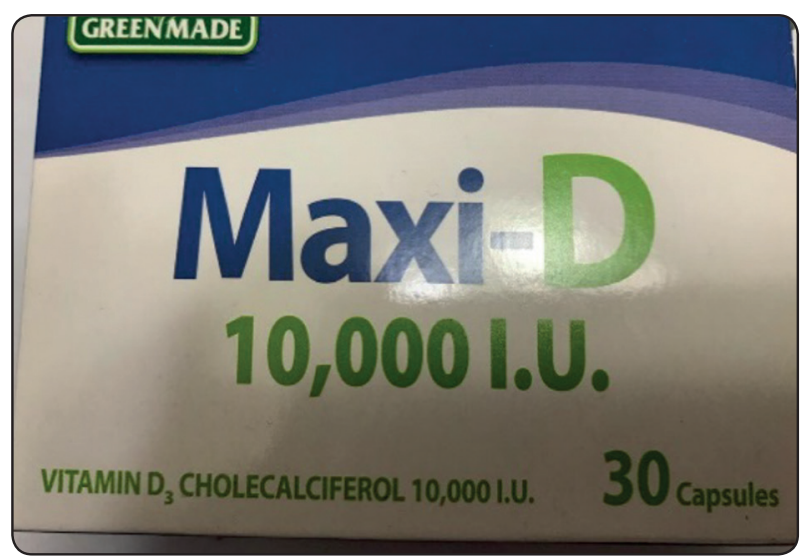

Fig. (1) 


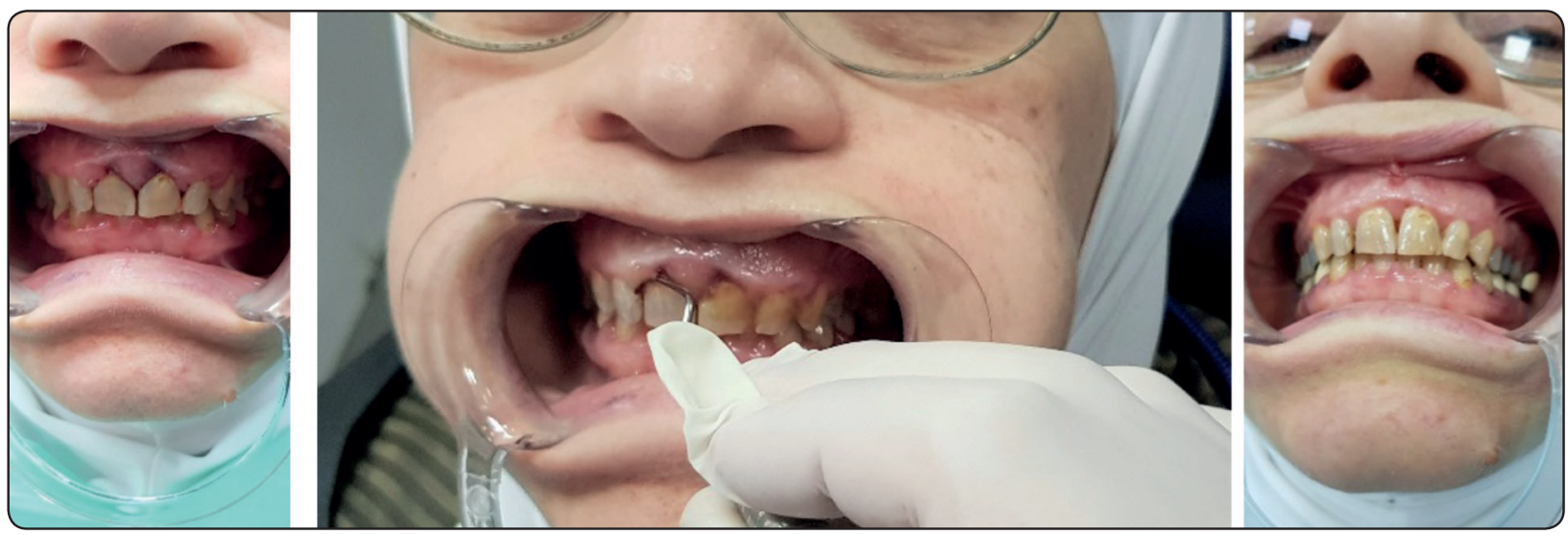

Fig. (2) Gingival inflammation with marked edema and spontaneous bleeding of 44 year female patient

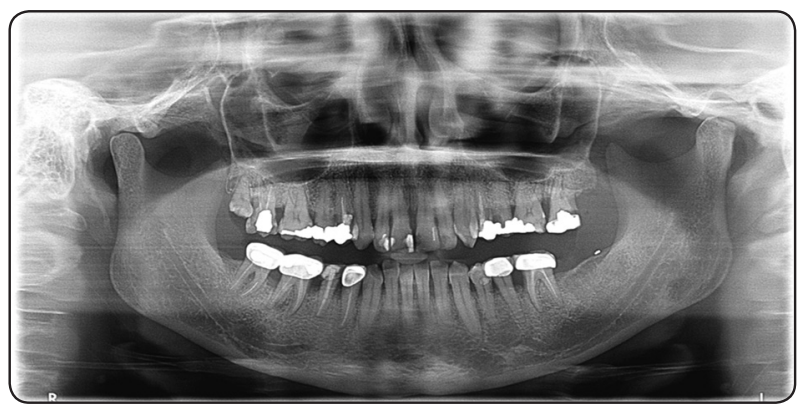

Fig. (5) Panoramic x-ray of the above female patient showing generalized mild and moderate bone loss

\section{Statistical Analysis}

All data were collected and analyzed using the Statistical Package Version 23.

Descriptive statistics were presented as frequency and percent for qualitative variables and mean and standard deviation for quantitative variables. The student t-test was used to compare the mean changes in the clinical parameters of two independent groups. Significance level was set at $5 \%$.

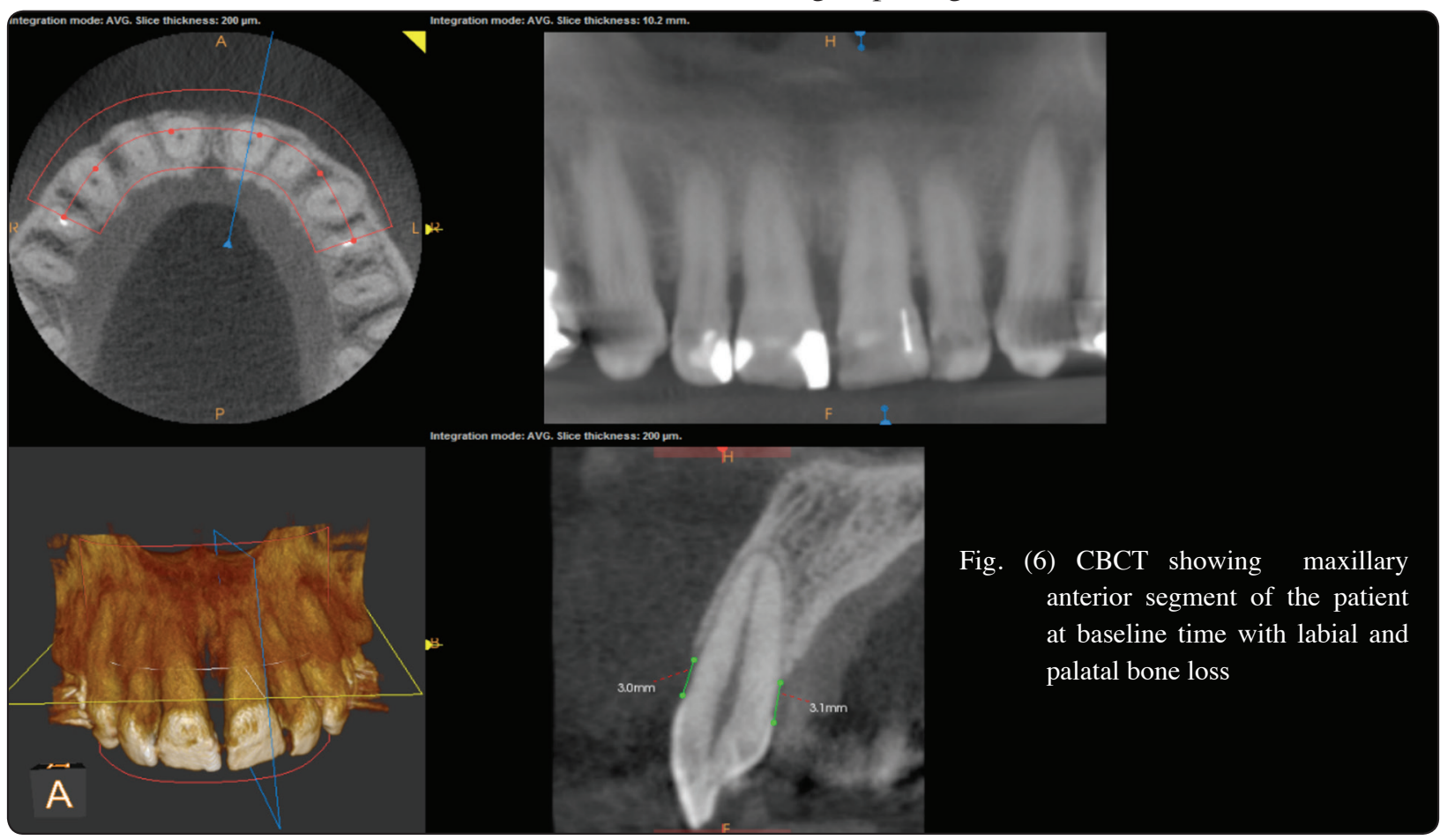




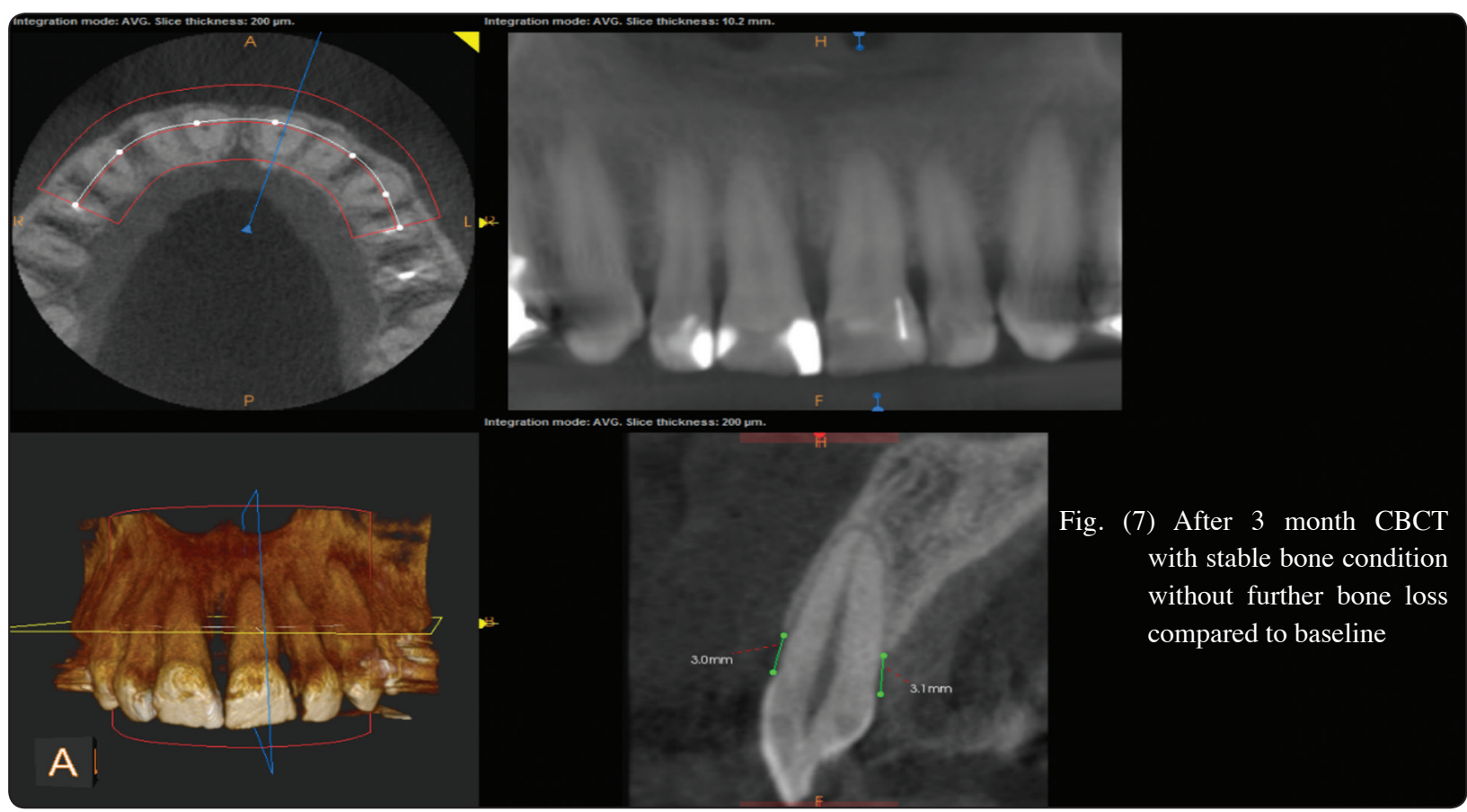

\section{RESULTS}

A total of 47 patients were assessed for eligibility for inclusion in this study. Up to 19 patients were excluded. 16 patients did not meet the inclusion criteria and 3 patients refused to participate. A total number of 28 patients were included in the study after obtaining their informed consent and were randomized and allocated to either a test group or a placebo group of 14 participants each. There was no loss to follow up. All 28 participants were included in the final analysis (Figure 8).

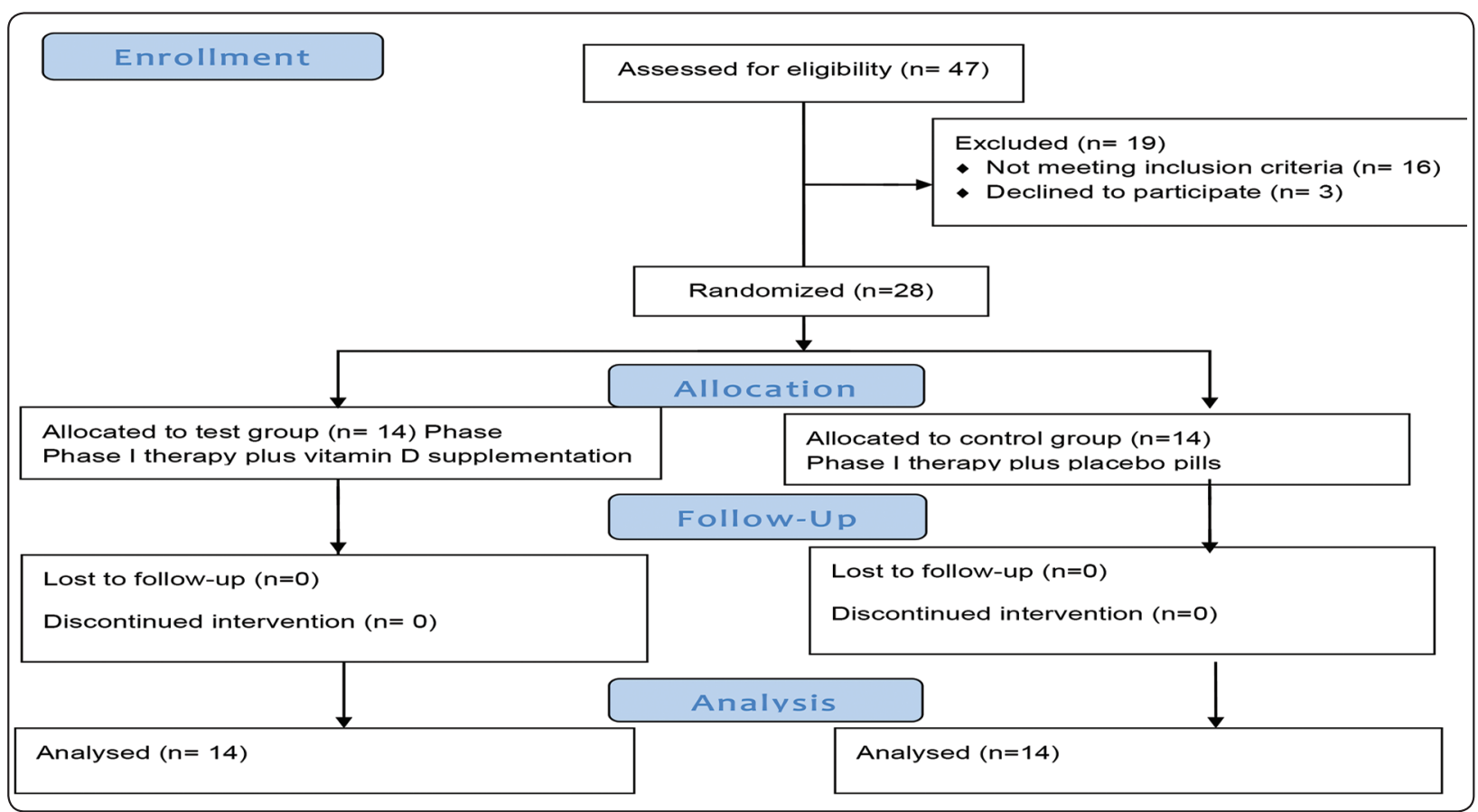

Fig. (8) CONSORT Flow Diagram 


\section{Description of the Groups:}

\section{Control Group:}

The control group consisted of 14 patients, aged between 30-45, 7 of which are females and 7 of which are males. All 14 patients are smokers, and a mean Vitamin D blood level of 17.36 at baseline (Table 1). Scaling and Root Planing was performed however, this group was not given a Vitamin D supplement.

\section{Test Group:}

The test group consisted of 14 patients, also aged 30-45 and consisted of 7 males and 7 females. All 14 patients were smokers with a mean Vitamin D blood level of 14.36 at baseline. The mean Vitamin D blood level after two month was 37.64 (Table 1). This group was given a vitamin D supplement in addition to scaling and root planing.

There was an increase by 23.28 units in the mean Vitamin D Blood level for the Test group after three months of Vitamin D supplementation (Table 1).

\section{Percent Change in Gingival Indices among Each Group from Baseline to Three Months after Completion of the Protocol:}

\section{Control Group:}

The control group had a $14.7 \%$ decrease in the mean gingival index from 0.9536 at baseline to 0.8064 after three months, a $19.5 \%$ decrease in the mean plaque index from 1.0629 at baseline to 0.8679 after three months, $18.93 \%$ decrease in the mean probing pocket depth from 3.1986 at baseline to 3.0093 after three months and a $19.5 \%$ decrease in the mean clinical attachment level from 2.3079 at baseline to 2.1129 after three months (Table 2).

\section{Test Group:}

The test group had a $33.35 \%$ decrease in the mean gingival index from 1.1821at baseline to 0.8486 after three months, a $29.43 \%$ decrease in the mean plaque index from 0.8814 at baseline to 0.5871 after three months, a $45 \%$ decrease in the mean probing pocket depth from 2.2714 at baseline to 1.8214 after three months and a $32.71 \%$ decrease in the mean clinical attachment level from 1.9250 at baseline to 1.5979 after three months (Table 3).

\section{Comparing Percent Change in Gingival Indices between the Control and Test Group from Base- line to Three Months after Completion of the Protocol:}

Independent $t$ test used to compare the changes in the mean periodontal indices between groups showed that the mean changes across all periodontal indices between groups were statistically significant at $p<0.05$. The change in the mean gingival index between groups was significant at $\mathrm{p}=0.000$; the change in the mean plaque index between groups was significant at $p=0.002$; the change in the mean probing pocket depth between groups was significant at $p=0.000$; the change in the mean clinical attachment level between groups was significant at $\mathrm{p}=0.001$ (Table 4).

The highest significant difference between the test group and the control group was in the probing pocket depth, where the test group had a $26.07 \%$ greater decrease than the control group. This is the only circumstance where being in the test group made a difference almost twice as large as being in the control group. The test group had greater differences in the decrease of all other indices: gingival index, mean clinical attachment level and plaque index.

The findings strongly suggest a strong association between Vitamin D supplementation and better gingival outcomes, among Vitamin D deficient middle aged healthy patients with mild to moderate chronic periodontitis, as an adjunctive treatment to the standard scaling and root planing. 
TABLE (1) Demographics:

\begin{tabular}{|l|c|c|}
\hline \multicolumn{1}{|c|}{ Variables } & $\begin{array}{c}\text { Control Group } \\
(\mathrm{n}=14)\end{array}$ & $\begin{array}{c}\text { Test Group } \\
(\mathrm{n}=14)\end{array}$ \\
\hline Age in years (mean) $\pm \mathrm{SD}$ & $36.14 \pm 4.975$ & $38.29 \pm 5.030$ \\
\hline Vitamin D Blood Level at Baseline $(\mathrm{ng} / \mathrm{ml}) \pm \mathrm{SD}$ & $17.36 \pm 5.930$ & $14.36 \pm 8.308$ \\
\hline Vitamin D Blood Level After Three Months $(\mathrm{ng} / \mathrm{ml}) \pm \mathrm{SD}$ & - & $37.64 \pm 5.153$ \\
\hline
\end{tabular}

TABLE (2) Mean Periodontal Parameters for the Control Group at Baseline and at Three Months and the percent change over time:

\begin{tabular}{|l|c|c|c|}
\hline \multicolumn{1}{|c|}{ Parameter } & Measurement at baseline & Measurement at three months & Percent change \\
\hline Mean Gingival Index & 0.9536 & 0.8064 & 14.7 \\
\hline Mean Plaque Index & 1.0629 & 0.8679 & 19.5 \\
\hline Mean Probing Pocket Depth & 3.1986 & 3.0093 & 18.9 \\
\hline Mean Clinical Attachment Level & 2.3079 & 2.1129 & 19.5 \\
\hline
\end{tabular}

TABLE (3) Mean Periodontal Parameters for the Test Group at Baseline and at Three Months and the percent change over time:

\begin{tabular}{|c|c|c|c|}
\hline Parameter & Measurement at baseline & Measurement at three months & Percent change \\
\hline Mean Gingival Index & 1.1821 & 0.8486 & 33.35 \\
\hline Mean Plaque Index & 0.8814 & 0.5871 & 29.43 \\
\hline Mean Probing Pocket Depth & 2.2714 & 1.8214 & 45.00 \\
\hline Mean Clinical Attachment Level & 1.9250 & 1.5979 & 32.71 \\
\hline
\end{tabular}

TABLE (4) Comparison of Mean Change in Gingival Indices between the Control and Test Group from Baseline to Three Months after Completion of the Protocol (negative percentages indicate better periodontal health):

\begin{tabular}{|c|c|c|c|c|c|}
\hline \multicolumn{7}{|c|}{ Independent t-test } \\
\hline & $\mathrm{t}$ & Sig. (2-tailed) & Mean Difference & Percent Diff. & Std. Error Diff. \\
\hline Change in Mean Gingival Index & -5.293 & $.000^{*}$ & -.18643 & -18.65 & 0.03522 \\
\hline Change in Mean Plaque Index & -3.476 & $.002^{*}$ & -.09929 & -9.93 & 0.02856 \\
\hline Change in Mean Probing Pocket Depth & -9.663 & $.000^{*}$ & -.26071 & -26.07 & 0.02698 \\
\hline Change in Mean Clinical Attachment Level & -3.727 & $.001^{*}$ & -.13214 & -13.21 & 0.03546 \\
\hline
\end{tabular}

*Indicates threshold for statistical significance was set at $P<0.05$ 


\section{DISCUSSION}

There is limited data in the literature that evaluate the effect of Vitamin D supplementation alone on periodontal indices in middle aged Vitamin $\mathrm{D}$ deficient healthy patients with mild to moderate chronic periodontitis. Our results indicate that adjunctive Vitamin D supplementation in addition to standard scaling and root planning in mild to moderate chronic periodontitis in middle aged Vitamin D deficient healthy patients significantly improves periodontal indices measures including the mean gingival index, the mean plaque index, the mean probing pocket depth and the mean clinical attachment level compared to scaling and root planning alone.

Our results are consistent with the findings in the literature that show a trend of improved periodontal indices after Vitamin D supplementation. However, all the research studies conducted so far have used Vitamin D with calcium with chronic periodontitis patients in contrast with our study that used exclusively Vitamin D supplementation to test its sole effectiveness on periodontal indices as an adjunctive treatment to standard scaling and root planning in Vitamin D deficient patients.

The research studies conducted so far have recruited patients with advanced aged groups who may be more likely at risk of osteoporosis and alveolar bone loss and therefore may benefit from calcium supplementation in addition to Vitamin D, since the positive effects of calcium and vitamin D supplementation on bone health has been well documented in the literature and for a lesser extent for alveolar bone loss (10-12) Therefore, in this study we opted to supplement our patients with Vitamin D alone since they were in the middle aged group (30 to 45 years) and were less likely to have bone loss and may well obtain calcium from dietary sources such as dairy products or green vegetables.

The trend of improved periodontal indices after Vitamin D supplementation was shown in a cohort of patients who were followed over one year after supplementation with Vitamin D ( $\geq 400$ international units/day) and Calcium ( $\geq 1000 \mathrm{mg} /$ day). The cohort had shallower probing depths, fewer bleeding on probing sites and lower gingival index values. Moreover, fewer furcation involvements, less attachment loss and less alveolar crest height loss. After adjusting for covariates, the effect of vitamin D and calcium supplementation was statistically significant at baseline, borderline at 6 months and not statistically significant at 12 months $(\mathrm{p}=0.142)$. The authors reported that the non-significant effect of vitamin D and calcium supplementation at one year may be attributed to the relatively low dose of Vitamin D supplemented with a daily mean of 1049 IU (781-1317) and therefore, the dose was likely not sufficient to normalize serum $25(\mathrm{OH}) \mathrm{D}^{(11)}$. The authors suggested that a higher dose would have given more pronounced results. In fact, it has been recommended that doses as large as 3,800 to 5,000 IU/day of vitamin D3 are essential to ensure that vitamin $\mathrm{D}$ deficiency or insufficiency is resolved in about $80 \%$ of supplemented people ${ }^{(12)}$.

Of particular importance is that this study did not draw blood levels and document Vitamin D deficient status in the takers group since the authors assumed that a large proportion of the population is vitamin $\mathrm{D}$ deficient and assumed that their research subjects would also be Vitamin D deficient. However, in our study we drew blood levels from all our research subjects to objectively document the Vitamin D deficient status of the study participants. In addition, our subjects were taking a high dosage of Vitamin D supplementation according to accepted standards of care approved for Vitamin D deficient patients which explains the significant results that we obtained in all periodontal indices .

In a similar clinical trial, the test group was supplemented with a combined dose of $500 \mathrm{mg}$ calcium and 250 IU Vitamin D once daily for three months in addition to scaling and root planing. 
A total of 82 subjects who were recruited within the age group $\geq 35$ and $\leq 55$ years and had their blood drawn for vitamin D level and were included in the study when they were vitamin D deficient. Intergroup comparison of clinical parameters at the end of three months showed that there was statistically significant difference $(p<0.05)$, in the oral hygiene index-simplified, the gingival index and bone density but in case of probing pocket depth and the clinical attachment level $(\mathrm{p}>0.05)$ there was no statistically significant difference ${ }^{(8)}$. The nonstatistical significance in the latter measurements was most due to the low dose of vitamin D the patients received as explained by the authors. In fact, current guidelines propose a dose of 50,000 IU of vitamin D taken orally once weekly up to 3 months for treatment of Vitamin D deficiency after which a maintenance dose of 800 to $2000 \mathrm{IU}$ is needed to avoid recurrent deficiency ${ }^{(21)}$

The dose response relationship was also demonstrated in another study by Hiremath et al (19). To evaluate the anti-inflammatory effect of vitamin $\mathrm{D}$ on gingivitis at various doses, oral vitamin $\mathrm{D}$ supplementation was given in three groups for three months period. Group A took doses of 2000 IU , 1000 IU for group B, 500 IU for group C and a placebo for group D. The study findings showed that the significant anti-inflammatory effect was seen in the high vitamin D dose group A after one month, in the moderate vitamin $\mathrm{D}$ dose group $\mathrm{B}$ at two months and in the low vitamin D dose group $\mathrm{C}$ at three month time interval after vitamin $\mathrm{D}$ supplementation $(\mathrm{P}<0.0001)$ while the placebo group did not show any significance.

Improved periodontal outcomes with adjunctive Vitamin D can be explained though several mechanisms (16,18,22). Vitamin D has been postulated to have anti-inflammatory effects by reducing the likelihood of developing gingival inflammation ${ }^{(7,11)}$. While standard scaling and root planing may not completely eliminate oral bacterial pathogens, vitamin D supplementation may prove to be a safe adjunctive treatment to standard periodontal therapy. Also, it proposed to have a broad anti-bacterial spectrum in fighting harmful bacteria associated with periodontal disease since bacteria do not develop resistance to its antimicrobial peptides such as LL-37 present in vitamin $\mathrm{D}^{(24)}$

Vitamin D could improve periodontal outcomes by slowing the bone loss from various skeletal sites including alveolar bone and preventing tooth loss. Vitamin D increases the ability of the small intestine to absorb dietary calcium and phosphate. By enhancing intestinal uptake of both calcium and phosphate, vitamin D helps to create optimum conditions for bone mineralization (20,21). A recent systematic review investigated the effect of vitamin D level on periodontal treatment outcomes and reported that vitamin D deficiency at the time of periodontal treatment negatively affects treatment outcomes ${ }^{(15)}$.

Despite the small sample size recruited that was restricted to middle aged patients and the short follow up period, the patient allocation to the test and control group was random and double blinded and each group had equal number of both genders. All of the participants were smokers and relatively healthy which prevents the potential bias. Supplementation with vitamin $\mathrm{D}$ was only used and its sole effect was evaluated in comparison to other studies that combine calcium and vitamin D in one pill which can make it hard to determine the effect of Vitamin D alone on periodontal outcomes. However, since our research subjects were relatively young and healthy (30-45 years), we assumed that their risk of bone loss is low and dietary calcium intake was enough from dairy or vegetable sources. However, further long term research with a broader aspect of patient populations is needed to study the effect of Vitamin D supplementation on alveolar bone density and its relation with chronic periodontitis. 


\section{CONCLUSION}

We recommend that dentists who are performing standard periodontal therapy on patients with chronic periodontitis to measure the blood level of vitamin $\mathrm{D}$ in their patients. In vitamin D deficient patients, it is recommended to supplement their patients with vitamin $\mathrm{D}$ according to standards of care and that is 50,000 UI per week for 12 weeks or 10,000 UI five times weekly for 12 weeks followed by maintenance therapy of 50,000 UI per month. Vitamin D constitutes an inexpensive, safe and probably may contribute to decrease health care costs as an adjunctive therapy to standard periodontal treatment.

\section{REFERENCES}

1. Savage A, Eaton KA, Moles DR, Needleman I. A systematic review of definitions of periodontitis and methods that have been used to identify this disease. J Clin Periodontol. 2009 Jun;36(6):458-67.

2.Stein SH, Tipton DA. J Tenn Dent Assoc. Vitamin D and its impact on oral health-an update. 2011 Spring;91(2):30-3; quiz 34-5

3.Anand N, Chandrasekaran SC, Rajput NS. Vitamin D and periodontal health: Current concepts. J Indian Soc Periodontol. 2013;17(3):302-308.

4. Krishnan AV, Feldman D. Mechanisms of the anti-cancer and anti-inflammatory actions of vitamin D. Annu Rev Pharmacol Toxicol. 2011;51:311-36.

5. Arabi A, El Rassi R, El-Hajj Fuleihan G. Hypovitaminosis $\mathrm{D}$ in developing countries-prevalence, risk factors and outcomes. Nat Rev Endocrinol. 2010 Oct;6(10):550.

6. Dietrich T, Joshipura KJ, Dawson-Hughes B, BischoffFerrari HA. Association between serum concentrations of 25-hydroxyvitamin D3 and periodontal disease in the US population. Am J Clin Nutr. 2004 Jul;80(1):108-13.

7. Dietrich T, Nunn M, Dawson-Hughes B, Bischoff-Ferrari HA. Association between serum concentrations of 25-hydroxyvitamin D and gingival inflammation. Am J Clin Nutr. 2005 Sep;82(3):575-80.

8. Perayil J, Menon KS, Kurup S, Thomas AE, Fenol A, Vyloppillil R, Bhaskar A, Megha S. Influence of Vitamin
D \& Calcium Supplementation in the Management of Periodontitis. Journal of Clinical and Diagnostic Research. 2015 Jun, Vol-9(6): ZC35-ZC38

9.Papapanou PN, Sanz M, Buduneli N, Dietrich T, Feres M, Fine $\mathrm{DH}$, et al,. Periodontitis: Consensus report of workgroup 2 of the 2017 World Workshop on the Classification of Periodontal and Peri-Implant Diseases and Conditions. J Periodontol. 2018 Jun;89 Suppl 1:S173-S182.

10. Jagelavičienė E, Vaitkevičienė I, Šilingaitė D, Šinkūnaitė E, Daugèlaitè G. The Relationship between Vitamin D and Periodontal Pathology. Medicina (Kaunas). 2018 Jun $12 ; 54(3)$.

11. Hildebolt CF, Pilgram TK, Dotson M, Armamento-Villareal R, Hauser J, Cohen S, Civitelli R. Estrogen and/or calcium plus vitamin $\mathrm{D}$ increase mandibular bone mass. $\mathrm{J}$ Periodontol. 2004 Jun;75(6):811-6.

12. Ramesh A, Mahajan K, Thomas B, Shenoy N, Bhandary R. Alveolar bone mass in pre- and postmenopausal women with serum calcium as a marker: a comparative study. Indian J Dent Res. 2011 Nov-Dec;22(6):878

13. Garcia MN, Hildebolt CF, Miley DD, Dixon, Couture RA, Spearie CL, Langenwalter EM, Shannon WD, Deych E, Mueller C, Civitelli R. One-year effects of vitamin D and calcium supplementation on chronic periodontitis. $\mathrm{J}$ Periodontol. 2011 Jan;82(1):25-32.

14. Aloia JF, Patel M, Dimaano R, Li-Ng M, Talwar SA, Mikhail M, Pollack S, Yeh JK. Vitamin D intake to attain a desired serum 25-hydroxyvitamin D concentration. Am J Clin Nutr. 2008 Jun; 87(6):1952-8.

15. Fakheran O, Khodadadi-Bohlouli Z, Khademi A. Effect of vitamin D level on periodontal treatment outcomes: a systematic review. Gen Dent. 2019 Mar-Apr;67(2):64-67.

16. Khammissa RAG, Ballyram R, Jadwat Y, Fourie J, Lemmer J, Feller L. Vitamin D Deficiency as It Relates to Oral Immunity and Chronic Periodontitis. Int J Dent. 2018 Oct 1;2018:7315797.

17. Al-Harthi LS, Cullinan MP, Leichter JW, Thomson WM. Periodontitis among adult populations in the Arab World. Int Dent J. 2013 Feb;63(1):7-11.

18. Alshouibi EN, Kaye EK, Cabral HJ, Leone CW, Garcia RI. Vitamin D and periodontal health in older men. J Dent Res. 2013 Aug;92(8):689-93

19. Hiremath VP, Rao CB, Naik V, Prasad KV. Antiinflammatory effect of vitamin $\mathrm{D}$ on gingivitis: a dose- 
response randomised control trial. Oral Health Prev Dent. 2013;11(1):61-9.

20. Jiao Y, Hasegawa M, Inohara N. Emerging roles of immunostimulatory oral bacteria in periodontitis development. Trends Microbiol. 2014 Mar;22(3):157-163.

21. Kennel KA, Drake MT, Hurley DL. Vitamin D deficiency in adults: when to test and how to treat. Mayo Clin Proc. 2010 Aug;85(8):752-7

22. Millen AE, Hovey KM, LaMonte MJ, Swanson M, Andrews CA, et al . Plasma 25-hydroxyvitamin D concentrations and periodontal disease in postmenopausal women. J
Periodontol. 2013 Sep;84(9):1243-56.

23. Miley DD, Garcia MN, Hildebolt CF, Shannon WD, Couture RA, Anderson ,et al. Cross-sectional study of vitamin $\mathrm{D}$ and calcium supplementation effects on chronic periodontitis. J Periodontol. 2009 Sep;80(9):1433-9.

24. McMahon L, Schwartz K, Yilmaz O, Brown E, Ryan LK, Diamond G. Vitamin D-mediated induction of innate immunity in gingival epithelial cells. Infect Immun. 2011 Jun;79(6):2250-6. 